\title{
Radiomics - the value of the numbers in present and future radiology
}

\author{
Mateusz Patyk ${ }^{1 A, B, C, D, E, F}$, Jurand Silicki ${ }^{1 A, B, C, D, E, F}$, Rafał Mazur ${ }^{1 D, E}$, Roksana Kręcichwost ${ }^{2 E}$, \\ Dąbrówka Sokołowska-Dąbek ${ }^{10, E}$, Urszula Zaleska-Dorobisz ${ }^{1 A, D, E}$
}

'Department of General and Paediatric Radiology, Wrocław Medical University, Wrocław, Poland

2Department and Clinic of Ophthalmology, Wrocław Medical University, Wrocław, Poland

\section{Abstract}

\begin{abstract}
Radiomics is a new concept that has been functioning in medicine for only a few years. This idea, created recently, relies on processing innumerable quantities of metadata acquired from every examination, followed by extraction thereof from relevant imaging examinations, such as computer tomography (CT), magnetic resonance imaging (MRI), or positron emission tomography (PET) images, by means of appropriate created algorithms. The extracted results have great potential and broad possibilities of application. Thanks to these we can verify efficiency of treatment, predict locations of metastases of tumours, correlate results with histopathological examinations, or define the type of cancer more precisely. In effect, we obtain more personalised treatment for each patient, which is extremely important and highly recommendable in the tests and applicable treatment therapies conducted nowadays. Radiomics is a non-invasive and high efficiency post-processing method. This article is intended to explain the idea of radiomics, the mechanisms of data acquisition, existing possibilities, and the challenges incurred by radiologists and physicians at the stage of making diagnosis or conducting treatment.
\end{abstract}

Key words: radiomics, biomarkers, treatment response, quantitative imaging, segmentation, image features, precision medicine, informatics, machine learning.

\section{Introduction}

Contemporary medicine is characterised by the search of objective data, parameters, and factors underlying many diseases, particularly cancers or autoimmunological diseases (Lavelle et al. 2015). Detailed knowledge of the genetic code and the development of genomic science allow to us discover mutations characteristic of many pathologies, e.g. breast cancer in patients with the BRCA-1 mutation [1]. The discovery of the basic processes occurring in sick cells allowed the invention of biological medications that modify the metabolic pathways in autoimmunological diseases, e.g. TNF- $\alpha$ inhibitors in the treatment of rheumatic diseases [2]. Various types of proteins, cytokines, genetic mutations, etc. are so-called biomarkers, which permit the prediction of the likelihood of occurrence of a disease, its course (all those at a high rate of probability), and define its prognosis [3]. Imaging diagnostics are an indispensable tool, providing a lot of information about the patient's state and the disease [4].

In oncology, neurology, or autoimmunological diseases, modern imaging technologies such as computer tomography (CT), magnetic resonance imaging (MRI), positron emission tomography (PET), scintigraphy, digital radiography (CXR), and ultrasonography (US) allow us to accurately define the occurrence and assess the degree of advancement of anomalies [4]. The images obtained are interpreted by qualified personnel, and the accuracy of their assessment depends on the knowledge possessed, experience, and the quality of equipment and advanced post-processing software. Medical images, stored as digital files, are not only a set of images that can be "viewed

Correspondence address:

Mateusz Patyk, Department of General and Paediatric Radiology, Wrocław Medical University, 68 M. Curie-Skłodowskiej St., 50-369 Wrocław, Poland,

phone: +48 717842651 , e-mail: mtpatyk@gmail.com

Authors' contribution:

A Study design · B Data collection · C Statistical analysis · D Data interpretation · E Manuscript preparation · F Literature search · G Funds collection 
and interpreted", but they also form a vast resource of biomedical data saved in the form of a complex numerical code [5]. These data include hundreds, or sometimes even thousands, of parameters - features unique to selected pathologies [6].

For the first time, specific parameters were used in computer-aided detection and diagnosis software (CAD), amongst others in breast cancer, lung tumour diagnostics, or in automatic spine segmentation systems [7]. Currently, these applications are the basic furnishing of packages e.g. for assessment of focal changes in lungs or liver, standardised according to the RECIST 1.1 protocol [8].

Discoveries at the level of basic science, which initiated the creation of specific areas such as proteomics, genomics, and metabolomics, inspired the search for image biomarkers, characteristic features for selected disease entities [9]. The term radiomics was first introduced in 2010; it is derived from the name of the sector (radiology) and a characteristic suffix found in the fields of basic sciences (omics) $[10,11]$. Since that time, we have observed a significant growth of interest in quantitative imaging biomarkers.

The aim of this article is to review the available methods of advanced post-processing and to approximate the results of the examinations using contemporary radiomics.

This systematic review of the literature was conducted based on the PubMed, Clinical Key, and ScienceDirect databases, using the following key words: radiomic or radiomics. The analysis covered the works developed between January 2007 and July 2017, available in the form of full articles written in English. Out of 225 articles found, the 15 most recent publications were used in further analysis; these were also the most extensive and included the most information, which allowed a reliable approximation of the subject of radiomics and its possibilities.

\section{Radiomics}

Radiomic assessment of images is a complex process, in which at least five major stages should be highlighted: acquisition of medical images, selection of the region of interest (ROI), three-dimensional modelling and segmentation of the region, analysis of features, and finally integration with the results of other assessments (clinical, laboratory data, or genetic tests) [5]. The key for each stage is to follow the standards and the adopted algorithms in order to be able to reproduce the achieved effect and to implement it in other healthcare centres.

\section{Medical imaging}

The basis for radiomics is CT and MR examinations, while PET and more advanced PET/CT or PET/MR to a lesser extent [12]. Although acquisition of good quality, unified imaging data does not constitute any problem in everyday clinical practice, it is a significant challenge in terms of cooperation between individual healthcare centres [5].
Manufacturers of radiological equipment apply diverse techniques for image acquisition, reduction of radiation, or post-processing techniques, which may affect the image parameters and in consequence may give various results of radiomic analysis.

\section{Region of interest and $3 \mathrm{D}$ rendering}

Determination of the region of interest allows us to extract that fragment of the image from the entire examination i.e. a virtual tissue - which is affected by the disease. A perfect example is the selective choice of the tumour region in lung cancer [13]. For this purpose, manual and, currently more frequently, automatic programmes of change segmentation are used - CAD. Numerous studies confirm the credibility and high reproducibility of automatic and semi-automatic diagnosis-aiding programmes [1-16]. These programmes recognise the density and structure of the tissue and "resect" it from the healthy tissues [17]. That is followed by $3 \mathrm{D}$ models, which work as a material for further analysis of features.

\section{Radiomic features}

Radiomics enables quantitative isolation of image features, which may be grouped within several cognitive and statistical levels [5,12]. The first level, which comprises semantic features (shape, size, location of abnormality, degree of its vascularisation, spicularity, infiltration of the region), which are used in everyday clinical practice [6]. The second level is associated with quantitative parameters of each voxel the smallest volumetric units in three-dimensional images [12]. It presents the distribution of numerical values of individual voxels in the form of a histogram, returning such parameters as the average, maximum, minimum, standard deviation, kurtosis, obliquity, entropy, etc. The third level, statistical analysis of the higher order structure, shows the relationships (similarities and counterparts) between the neighbouring voxels. Image structure analysis was first proposed in 1973 by Haralick et al. in the study: Textural features for image classification [18].

Dozens of various image texture analysis methods are available currently, of which radiomics uses the following: the grey-level co-occurrence matrix (GLCM), grey-level run length matrix (GLRLM), grey-level size zone matrix (GLSZM), neighbourhood grey-tone difference matrix (NGTDM), fractal features analysis and discrete wavelet transform (DWT), Minkowski functionals, and Laplace transforms [5].

GLCM defines the relationships between voxel pairs and the frequency of occurrence of similar pairs in ROI [12]. GLRLM returns the quantity of adjacent voxels with the equal level of grey [19]. GLSZM refers to the level of ROI uniformity [6]. NGTDM is a mono-dimensional measure of differences between all grey values of individual pixels [6]. NGTD defines the contrast, roughness, complexity, and 
texture strength. Fractals characterise self-resemblance and roughness of the surface lesion. The list of statistical transformations and capabilities to distinguish image features seems to be indefinite, particularly in combination with the abundance of imaging techniques and the values specific to the selected pathologies. Another method of extraction of desirable information is the use of spatial filters; for example, edge filtering techniques, Laplacian of Gaussian filter, entropy filter etc. [12]. A detailed description of individual techniques goes far beyond the scope of this work.

\section{Data integration}

The last stage of this complex process is integration of data acquired from other sources, amongst others: clinical data, laboratory results, presence of antibodies, and specific proteins [20]. The creation of enormous databases and application of multi-factorial statistical analyses is intended to facilitate diagnosis of diseases, establish prognosis, and, as a further consequence, to help in the creation of targeted screening programmes or medications preventing illness [21].

\section{Current tests}

The radiomics field is an extremely rapidly developing area of contemporary medicine, which penetrates many of its sectors and closely related sciences. The database of scientific works is continuously increasing, while radiomics is dynamically conquering new areas. This part of the article will present the most important research and the latest overview of publications put forward in 20162017 in the field of oncology, which is the most developed and represented domain of medicine in reviews of articles conducted on a large number of patients (at least 100), which will allow objective results to be obtained. The selected literature refers to the tests conducted using computer tomography - a method that currently features the most extensive resource of data. In the study conducted on a group of 127 patients who were analysed for pathological response during neo-adjuvant chemotherapy within the course of NSCLS treatment [22], a radiomic predictive value of distinct features was observed, contrary to classical imaging.

In the next study conducted on a group of $200 \mathrm{pa}-$ tients, who were examined with respect to prediction of metastases to lymph nodes in the course of colorectal cancer [23], it was also possible to achieve success and to create an adequate model (normogram), which pre-operatively facilitates prediction of occupation of specific lymph nodes in each patient individually.

A study associated with EGFR mutation status in lung adenocarcinoma, conducted on 298 patients, allowed the presence of mutations to be foreseen, by means of designing of a model analysing five features, together with a summary of the risk factors. With such a normogram, there is tremendous potential to improve the differentiation of EGFR type from wild type [24].

In research concerning HNSC, which was based on two groups consisting of 101 and 95 subjects respectively, it was possible to characterise prognostic factors and adequate methods of data acquisition in order to achieve a final estimate of life expectancy of patients with head and neck cancer. The results achieved allow precise planning of treatment [25].

In retrospective research conducted on a group of 215 patients after partial hepatectomy due to hepatocellular cancer (HCC), data were collected, on the basis of which an appropriate algorithm was designed. The model was extended with additional parameters such as clinical factors, which alone do not give a precise response on HCC recurrence. As a result, a tool was found that enables discovery of early recurrence of the disease much earlier than relying solely on clinical data [13].

\section{Hopes and concerns}

A challenge for $21^{\text {st }}$ century medicine is the collection of great amounts of information about patients and diseases, across numerous databases and reports, referred to as Big Data [26,27]. Creators of the largest programmes, launched amongst others in the USA, the Netherlands, and France, emphasise the main limitation: lack of coherence of data originating from different institutions [5]. Implementation of standardised protocols is an extremely important element, which will allow the extraction of representative, reliable, and, most of all, repeatable results [12]. The creation of common, coherent databases is one of the greatest challenges faced by contemporary medicine.

Synthesis of data obtained from standard clinical tests, histopathological, genetic, and, ultimately, radiological results will allow the development of more personalised medicine [26]. Discovery of the rules and patterns encoded in images give hope for better recognition of the pathophysiology of cancers, amongst others, and their discovery at earlier stages, as well as for improvement of treatment efficiency. Use of radiological tests being non-invasive screening methods, in combination with radiomics, may enable a very early and precise diagnosis, and individualised patient care.

The enormous amount of information is also associated with a risk of overfitting and overlooking more general information. Over-concentration on numbers, parameters, and indicators may cause dehumanisation of medicine.

Sceptics of the $20^{\text {th }}$ century expected radiology to be over by the year 2020 [28]. The discovery of radiomics and use of advanced post-processing techniques is proof, which not only contradicts the decline of this domain but also indicates its uncapped potential for further development. 


\section{Conclusions}

Radiomics is a relatively new branch, allowing abundant new information on many diseases.

Use of radiomic analysis techniques in cancer, and haematological and autoimmunological diseases may help to make earlier relevant diagnosis, adjust the best available therapy, and improve the patient's quality of life.
Currently, an important challenge for this domain is to develop standardised clinical protocols, reproducible across various healthcare institutions.

\section{Conflict of interest}

The authors report no conflict of interest.
References

1. Chamberlain JS, Boehnke M, Frank TS, et al. BRCA1 maps proximal to D17S579 on chromosome 17q21 by genetic analysis. Am J Hum Genet 1993; 52: 792-798.

2. Smolen JS, Landewé R, Bijlsma J, et al. EULAR recommendations for the management of rheumatoid arthritis with synthetic and biological disease-modifying antirheumatic drugs: 2016 update. Ann Rheum Dis 2017; 76: 960-977.

3. Enewold L, Mechanic LE, Bowman ED, et al. Serum concentrations of cytokines and lung cancer survival in African Americans and Caucasians. Cancer Epidemiol Biomarkers Prev 2009; 18: 215-222.

4. Giardino A, Gupta S, Olson E, et al. Role of Imaging in the Era of Precision Medicine. Acad Radiol 2017; 24: 639-649.

5. Gillies RJ, Kinahan PE, Hricak H. Radiomics: Images Are More than Pictures, They Are Data. Radiology 2015; 278: 563-577.

6. Avanzo M, Stancanello J, El Naqa I. Beyond imaging: The promise of radiomics. Phys Med 2017; 38: 122-139.

7. Tang J, Rangayyan RM, Xu J, et al. Computer-Aided Detection and Diagnosis of Breast Cancer With Mammography: Recent Advances. IEEE Trans Inf Technol Biomed 2009; 13: 236-251.

8. Eisenhauer EA, Therasse P, Bogaerts J, et al. New response evaluation criteria in solid tumours: Revised RECIST guideline (version 1.1). Eur J Cancer 2009; 45: 228-247.

9. Robles AI, Harris CC. Integration of multiple 'OMIC' biomarkers: A precision medicine strategy for lung cancer. Lung Cancer 2017; 107: 50-58.

10. Gillies RJ, Anderson AR, Gatenby RA, et al. The biology underlying molecular imaging in oncology: from genome to anatome and back again. Clin Radiol 2017; 65: 517-521.

11. Kumar V, Gu Y, Basu S, et al. NIH Public Access. Magn Reson Imaging 2013; 30: 1234-1248.

12. Parekh V, Jacobs MA. Radiomics: a new application from established techniques. Expert Rev Precis Med Drug Dev 2016; 1: 207-226.

13. Coroller TP, Grossmann P, Hou Y, et al. CT-based radiomic signature predicts distant metastasis in lung adenocarcinoma. Radiother Oncol 2015; 114: 345-350.

14. Castellino RA. Computer aided detection (CAD): An overview. Cancer Imaging 2005; 5: 17-19.

15. Hirose T, Nitta N, Shiraishi J, et al. Evaluation of Computer-aided Diagnosis (CAD) Software for the Detection of Lung Nodules on Multidetector Row Computed Tomography (MDCT). JAFROC
Study for the Improvement in Radiologists' Diagnostic Accuracy. Acad Radiol 2008; 15: 1505-1512.

16. Choi WJ, Choi TS. Automated pulmonary nodule detection system in computed tomography images: A hierarchical block classification approach. Entropy 2013; 15: 507-523.

17. Eadie LH, Taylor P, Gibson AP. A systematic review of computer-assisted diagnosis in diagnostic cancer imaging. Eur J Radiol 2012; 81: e70-76.

18. Haralick R, Shanmugan K, Dinstein I. Textural features for image classification. IEEE Trans. Syst. Man Cybern 1973; 3: 610-621.

19. Park BE, Jang WS, Yoo SK. Texture analysis of supraspinatus ultrasound image for computer aided diagnostic system. Healthc Inform Res 2016; 22: 299-304.

20. Lambin P, Rios-Velazquez E, Leijenaar R, et al. Radiomics: Extracting more information from medical images using advanced feature analysis. Eur J Cancer 2012; 48: 441-446.

21. Hassanpour S, Langlotz CP. Information extraction from multi-institutional radiology reports. Artif Intell Med 2016; 66: 29-39.

22. Coroller TP, Agrawal V, Narayan V, et al. Radiomic phenotype features predict pathological response in non-small cell lung cancer. Radiother Oncol 2017; 119: 480-486.

23. Huang YQ, Liang CH, He L, et al. Development and validation of a radiomics nomogram for preoperative prediction of lymph node metastasis in colorectal cancer. J Clin Oncol 2016; 34: 2157-2164.

24. Sacconi B, Anzidei M, Leonardi A, et al. Analysis of CT features and quantitative texture analysis in patients with lung adenocarcinoma: a correlation with EGFR mutations and survival rates. Clin Radiol 2017; 72: 443-450.

25. Parmar C, Grossmann P, Rietveld D, et al. Radiomic Machine-Learning Classifiers for Prognostic Biomarkers of Head and Neck Cancer. Front Oncol 2015; 5.

26. Lee G, Lee HY, Park H, et al. Radiomics and its emerging role in lung cancer research, imaging biomarkers and clinical management: State of the art. Eur J Radiol 2017; 86: 297-307.

27. Kim J. Big Data, Health Informatics, and the Future of Cardiovascular Medicine. J Am Coll Cardiol 2017; 69: 899-902.

28. Brink JA, Arenson RL, Grist TM, et al. Bits and bytes: the future of radiology lies in informatics and information technology. Eur Radiol 2017; 27: 3647-3651. 